\title{
Are patients at risk of malnutrition being identified in our hospital? Should all hospitals move to MUST (Malnutrition Universal Screening Tool)?
}

\author{
J. Rigby ${ }^{1}$ and F. C. Leslie ${ }^{2}$ \\ ${ }^{1}$ University of Keele and ${ }^{2}$ University Hospital of North Staffordshire (UHNS), Stoke-on-Trent, Staffordshire ST4 6QG, UK
}

The move to BMI-based screening tools and specifically MUST is very topical presently, but in our Trust, we have faced barriers to making a change. The aim of the study, as part of a medical student project, was to determine the prevalence of malnutrition risk using our current nutrition screening tool (NST) and MUST, to assess current screening and to establish whether patients are managed appropriately.

Sixty patients admitted to a large teaching hospital were prospectively assessed as part of a medical student project. We identified the five patients most recently admitted on 12 medical and surgical wards. The accuracy and compliance of completion of the NST was assessed. The NST is a 7-point tool incorporating factors e.g. age, weight loss, ability to eat, gut function and does not involve weight or height. Patients were also screened using MUST by a single individual (JR). Patients were reviewed 4 weeks later to establish length of hospital stay (LOS), mortality, any infections, wound healing problems and relevant nutritional information.

Of the 60 patients, 34 were female (56.6\%), median age was 73.5 years (range 17-93). In 20 patients height or weight could not be measured, and so mid-upper-arm circumference (MUAC) was used. Sixty-three percent of patients were 'at risk' of malnutrition using our NST and 57\% using MUST (moderate agreement, kappa 0.6), MUST identified more 'high-risk' patients but appeared less accurate for liver patients ( $80 \%$ at risk with UHNS tool $v .40 \%$ MUST). Only $58 \%$ of patients had been screened and $80 \%$ of those not screened were 'at risk' ( $20 \%$ high risk), i.e. $50 \%$ of 'at-risk' patients were not identified. There was significant variability of screening with three wards screening all patients and two wards screening 0 patients. Some wards traditionally thought to have low-screening rates scored $100 \%$ (orthopaedics), but generally rates for medical wards were better than on the surgical wards. Many patients (particularly on surgical wards) had received nutritional input including supplements but had not been screened and in $40 \%$ of the 'medium-risk' patients identified by screening, none of the appropriate management options e.g. food diary were in place.

Fifteen years after McWhirter and Pennington ${ }^{(1)}, 38 \%$ of patients had a BMI $\leq 20 \mathrm{~kg} / \mathrm{m}^{2}, 22 \%$ were obese, males $>$ females, $P=0.04$. Weight or MUAC was repeated in $62 \%$ of patients. $76 \%$ lost weight in hospital, mean BMI change $-2.7 \%$. 'At-risk' patients were more likely to develop infections, experience wound healing complications, have longer LOS $(P=0.005)$ and higher mortality rates. All mortalities (13\% of cohort) were identified 'at risk'. As expected, patients referred early to a dietician improved nutritionally and lost less weight, mean BMI change $+3.3 \%$.

Many malnourished or 'at-risk' patients are admitted to our Trust and improvements need to be made in recognition and management. Since the completion of this study, the data have been presented at Trust level and the need for better screening recognised. Since a third of the patients could not have BMI calculated, and needed MUAC, this is likely to have time and cost implications. The implementation of MUST would lead to approx. $30 \%$ increase in referrals to dietetics but given the evidence, we hope to get support to fund the change, particularly if we can show more appropriate targeting of input to those patients who need it.

1. McWhirter JP \& Pennington CR (1994) BMJ 308, 945-948 